Editorial

\title{
Cutting Edge Technologies by Silicon- and Silicon Oxide- Based Nanostructures
}

\author{
Francesca A. Scaramuzzo $\perp^{1},{ }^{1}$ Arántzazu González-Campo ${ }^{\circ},{ }^{2}$ and Alessandro Dell'Era ${ }^{1}$ \\ ${ }^{1}$ Department of Fundamental and Applied Sciences for Engineering, Sapienza University, Rome, Italy \\ ${ }^{2}$ Institut de Ciencia de Materials de Barcelona (ICMAB-CSIC), Barcelona, Spain \\ Correspondence should be addressed to Francesca A. Scaramuzzo; francesca.scaramuzzo@uniroma1.it
}

Received 31 October 2020; Accepted 31 October 2020; Published 10 February 2021

Copyright (C) 2021 Francesca A. Scaramuzzo et al. This is an open access article distributed under the Creative Commons Attribution License, which permits unrestricted use, distribution, and reproduction in any medium, provided the original work is properly cited.

Due to its semiconductive properties, silicon has been widely used and still represents the basis of electronics-related technologies. Despite having properties often very different from the pure element, silicon derivatives (e.g., $\mathrm{SiO}_{2}, \mathrm{SiC}$, and silanes) are widely exploited as well in a variety of fields, ranging from photonic to biomedicine. Besides the traditional applications, the wide spreading of nanostructures, with their enhanced and in some respect unexpected properties, is now opening new perspectives for the possible uses of silicon and its derivatives. In this thematic special issue, our purpose was to highlight cutting-edge technologies and most modern applications related to these materials, especially at the nanoscale. We are glad to announce that the five original research papers published here, which we will briefly present in the following, all perfectly fit with our starting aim.

Two of the papers focus on issues related to the crucial role of Si and its derivatives for nanoelectronic and nanophotonic applications, clarifying the synthetic aspects which can substantially improve the features of the final obtainable structures. In particular, R.B. Beck and K. Ber synthesized ultrathin silicon layers by the mean of plasma-enhanced chemical vapor deposition (PECVD), analysing the influence of each parameter (i.e., type of reactor, presence of specific gases, timeframe, and temperature) on the final product quality. The authors elucidated some of the effects and interactions between the studied process conditions, thus providing useful indications to achieve layers with the desired properties, especially in terms of crystallinity.

H.-K. Shin and coworkers focused on the electrical and microstructural properties of $\mathrm{Ni} / \mathrm{C}$-faced $4 \mathrm{H}-\mathrm{n}-\mathrm{SiC}$ sub- strates, analysing the crucial effect of temperature on ohmic contacts. Briefly, the authors were able to demonstrate that by optimizing the thermal annealing it is possible to obtain a vertically oriented NiSi phase allowing the formation of ohmic contacts; on the other hand, the horizontal-type NiSi phase obtainable at higher temperatures results in the degradation of ohmic behaviour.

Besides the electronic field, silicon and its derivatives are now recognized as useful intermediates for surface functionalization, nanostructure synthesis, and decoration. Within this context, Y. Wang and coworkers contributed to this special issue with two papers. The authors devoted their efforts to the development of rapid, efficient, and high-yield ways to obtain a new silane coupling agent, which was then fully characterized, also in terms of thermal stability. Being able to react at the same time with metal oxides and organic compounds, the synthesized thiohydrazide-iminopropyltriethoxysilane played a key role in the design and preparation of a drug carrier based on magnetic nanoparticles. As a proof of principle, the system was tested for loading doxorubicin and revealed excellent $\mathrm{pH}$ responsiveness for drug releasing.

Finally, within the field of Si for energy production and storage, P.P. Prosini and coworkers developed an efficient synthetic strategy for $\mathrm{Si}$ nanowires to be used as anodes in lithium-ion batteries. By growing Si nanostructures directly on the current collector, the authors were able at the same time (i) to overcome the problem of the volumetric expansion occurring in the anode during lithium alloying and (ii) to guarantee an excellent electric contact without adding conducting binders to the electrode. Despite the linear 
capacity fade observed upon cycling, at different values of the charging current, the synthetized nanowires showed an exceptionally high-rate capability.

Due to the importance of the topic and the relevance of the achieved results, we sincerely hope that the papers published in this special issue would be of high interest for all readers of the journal.

\section{Conflicts of Interest}

The Lead Guest Editor and the Guest Editors declare that there is no conflict of interest.

\section{Acknowledgments}

We thank all authors for their valuable work and the referees for their efforts in carefully reviewing the manuscripts.

Francesca A. Scaramuzzo Arántzazu González-Campo Alessandro Dell'Era 